\title{
Alcohol induced disorders
}

\author{
Peter Mikosch $\cdot$ Josef Marksteiner
}

Received: 15 December 2013 / Accepted: 17 December 2013 / Published online: 26 January 2014 (C) Springer-Verlag Wien 2013

Since prehistoric times ethanol $\left(\mathrm{C}_{6} \mathrm{H}_{5} \mathrm{OH}\right)$, commonly referred as alcohol, has been used by humans as the intoxicating ingredient of alcoholic beverages. Dried residues on 9,000-year-old pottery found in late Stone Age village of Jiahu in northern China imply that already Neolithic people consumed alcoholic beverages [1]. Alcohol and the adverse effects of its excessive consumption were well-recognized and documented in ancient history, especially in Biblical writings [2-5]. The first clear evidence of distillation comes from Greek alchemists working in Alexandria in the first century ad [6].

Today, alcoholic beverages of varying alcoholic content are widely consumed across the world in different social and cultural settings. Alcoholism with all its detrimental health and social problems is a serious health problem in many countries. In Austria, alcohol consumption is one of the highest in the European Union $[7,8]$. The way of alcohol consumption differs widely in the world and is influenced by the historic and cultural background. A historic and cultural perspective may give a first approach to a better understanding of how alcohol consumption is implemented in our society and how it is handled, if consumed extensively. Some cultures have integrated moderate alcohol consumption in daily living as part of the culture. As an example, light to moderate

OA Univ.-Doz. Dr. P. Mikosch, MD $(\bowtie)$

Ludwig Boltzmann Institute of Osteology at the Hanusch Hospital of WGKK and AUVA Trauma Centre Meidling,

1st Medical Department, Hanusch Hospital,

Heinrich Collin Str. 30, 1140 Vienna, Austria

e-mail: peter.mikosch@osteologie.at

Prim. Ao. Univ.-Prof. Dr. J. Marksteiner

Department of Psychiatry and Psychotherapy, LKH Hall in Tirol, Milserstr. 10, 6060 Hall in Tirol, Austria

e-mail: josef.marksteiner@tilak.at consumption of wine can be mentioned in countries of the Mediterranean Sea with Roman history (e.g., Italy, France, Spain, and Portugal). In these countries this kind of light alcohol consumption is part of the countries' cuisine and it has a long standing cultural history of integration into the way of living in these countries. On the contrary, in Northern Europe with a Germanic cultural background the drinking patterns are different with rather extensive drinking of beer or high-percentage alcoholic drinks. State driven programs to reduce alcohol consumption or even to ban the sale of alcoholics (e.g., Norway, Sweden, the United States in the 1930s) were of limited success. The most recent developments of drinking behavior, going back to this drinking pattern, is "binge drinking", mainly seen with young people. Apart from the cultural influences, religion also played a role concerning alcohol consumption. As an example, the Jewish religion allows some limited alcohol use at certain events and under fulfillment of specific criteria (kosher), whereas alcohol consumption is generally forbidden in Islam.

This special issue on alcohol will not deal on all aspects of alcohol and alcoholism; in this respect, the matter is too complex and broad to be dealt within one journal issue. However, some special topics will be covered in this special issue on alcohol:

Wieser and Tilg [9] will report on severe alcoholic hepatitis-a life threatening condition with mortality rates up to $50 \%$. Beside the pathophysiology of this condition, which is a good example of a disease promoted by proinflammatory cytokines, the clinical conditions and their management will be presented in this paper.

Cognitive impairments will be reviewed by Weiss et al. [10] separated in view of acute alcohol intoxication and chronic alcoholism. Apart from this, gender specific differences and social implications regarding cognitive deficits will also be discussed in this article. 
An organ, which is less known to be influenced by alcohol consumption, is bone. Mikosch [11] will present the interrelationship of alcohol and the skeletal system. Apart from the pathophysiology of bone changes induced by alcohol, clinical effects on bone, such as osteopenia/ osteoporosis including fractures and osteonecrosis will also be presented. Therapeutic options from the osteological point of view will finalize this article.

Finally, diagnosis and also the follow-up of patients with alcoholism is sometimes difficult in terms of quantifying the extent of abuse and whether or not the patient still continues or stopped drinking. Thon et al. [12] will present and discuss the role of ethanol metabolites as biological markers in the diagnosis and therapy of alcohol use disorders and will show up in which clinical settings the use of such markers will be beneficial.

At this point special thanks to all contributing authors who made this special issue on alcohol possible. We hope that with the articles presented on some aspects of alcoholism this topic will once again bring alcohol and alcoholism to our minds as a relevant and ongoing problem of our society and our health care system. We wish the readers of the $W M W$ enjoyment and new insights on alcohol with this issue.

\section{Conflict of interest}

The authors declare no conflict of interest.

\section{References}

1. McGovern PE, Zhang J, Tang J, et al. Fermented beverages of pre-and proto-historic China. PNAS. 2004;101:17593-8. doi:10.1073/pnas.0407921102.
2. Mathew VM. Alcoholism in Biblical prophecy. Alcohol Alcohol. 1992;27:89-90.

3. Kaplan I. The first recorded case of alcohol abuse-Noah's arcoholism. Md Med J. 1993;42:444-5.

4. Altschuler EL. Societal consequences of Noah's alcohol misuse. S Afr Med J. 2005;95:820.

5. Seller SC. Alcohol abuse in the New Testament. Alcohol Alcohol. 1987;22:83-90.

6. Forbes RJ. A short history of the art of distillation. Leiden. EJ Brill; 1970. p. 6.

7. Rathner G, Dunkel D. Incidence of alcoholism and problem drinking in Austria. Wien Klin Wochenschr. 1998;110:356-63.

8. Uhl A, Bachmayer S, Puhm A, et al. Handbuch Alkohol-Österreich Band 1: Formeln und Zahlen 2013. Bundesministerium für Gesundheit, 5. Auflage, vollständig überarbeitete Auflage. Wien;2013.

9. Wieser W, Tilg H. Management of severe alcoholic hepatitis. Wien Med Wochenschr. 2014;164. doi:10.1007/ s10354-013-0221-5.

10. Weiss E, Singewald E, Ruepp B, Marksteiner J. Alcohol induced cognitive deficits. Wien Med Wochenschr. 2014;164. doi:10.1007/s10354-013-0226-0.

11. Mikosch P. Alcohol and bone. Wien Med Wochenschr. 2014;164. doi:10.1007/s10354-013-0258-5.

12. Thon N, Weinmann $\mathrm{W}$, Yegles $\mathrm{M}$, et al. What ethanol metabolits as biological markers tell us about alcohol use. Wien Med Wochenschr. 2014;164. doi:10.1007/ s10354-013-0254-9. 